\title{
Cuidados de enfermagem ao paciente oncológico em tratamento quimioterápico ambulatorial
}

\author{
Nursing care given to cancer patients undergoing for outpatient chemotherapy \\ Cuidados de enfemería prestados a los pacientes oncológicos sometidos a quimioterapia \\ ambulatoria
}

Flávia dos Santos Lugão de Souza ${ }^{1 *}$, Ana Carolina Souza Abreu ${ }^{1}$, Darlem Aparecida Pio ${ }^{1}$, Heloise Mota de Paiva Vieira Sanglard ${ }^{1}$, Naira Agostini Rodrigues dos Santos ${ }^{1}$.

\section{RESUMO}

Objetivo: Descrever os estudos que abordam os cuidados de enfermagem aos pacientes oncológicos adultos em tratamento quimioterápico sistêmico ambulatorial. Material e Método: Revisão da literatura, nos idiomas português e inglês, utilizando bases de dados eletrônicas, realizada nos dias 15 de maio a 30 de junho de 2018. Resultados: O câncer abrange um grupo de mais de 100 doenças que possuem em comum a capacidade de crescimento irregular de suas células que acometem tecidos e órgãos. $O$ tipo de tratamento ocorre pelo tipo, local e grau específico do câncer. A quimioterapia consiste no uso de um ou mais compostos químicos (quimioterápicos) para o tratamento do câncer e de doenças causadas por agentes biológicos, podendo ser administrada concomitante à cirurgia e a radioterapia. Conclusão: É importante o enfermeiro ter conhecimento específico para atuar como educador em saúde e prestador da assistência ao paciente em tratamento quimioterápico ambulatorial e para promoção do relacionamento interpessoal que proporcionará ao paciente um enfrentamento do tratamento de maneira mais satisfatória. Essa educação, norteada pela capacidade de compreensão de cada indivíduo, promove o entendimento correto da doença, tratamento, o manejo dos efeitos colaterais e vivências dos pacientes.

Palavras-Chave: Antineoplásicos, Quimioterapia, Cuidados de Enfermagem.

\begin{abstract}
Objective: To describe the studies that address nursing care for adult oncology patients undergoing outpatient systemic chemotherapy. Material and Method: Review of the literature, in the Portuguese and English languages, using electronic databases, held from May 15 to June 30, 2018. Results: Cancer encompasses a group of more than 100 diseases that have in common the irregular growth capacity of their cells that affect tissues and organs. The type of treatment is given by the type, stage and specific degree of cancer. Chemotherapy consists of the use of one or more chemical compounds (chemotherapeutics) for the treatment of cancer and diseases caused by biological agents, and can be administered concomitantly with surgery and radiotherapy. Conclusion: It is important for the nurse to have specific knowledge to act as a health educator and patient care provider in outpatient chemotherapy treatment and to promote the interpersonal relationship that will provide the patient with a more satisfactory treatment response. This education, guided by the capacity of understanding of each individual, promotes the correct understanding of the disease, treatment, management of the side effects and experiences of the patients.
\end{abstract}

Key words: Antineoplastic Agents, Chemotherapy, Nursing Care.

${ }^{1}$ Faculdade do Futuro (FAF), Manhuaçu- MG. *E-mail: flavia.I.s@terra.com.br 


\section{RESUMEN}

Objetivo: Describir los estudios que abordan los cuidados de enfermería a los pacientes oncológicos adultos en tratamiento quimioterápico sistémico ambulatorial. Material y Método: Revisión de la literatura, en los idiomas portugués e inglés, utilizando bases de datos electrónicas, celebrada del 15 de mayo al 30 de junio de 2018. Resultados: El cáncer abarca un grupo de más de 100 enfermedades que poseen en común la capacidad de crecimiento irregular de sus células que afectan a los tejidos y los órganos. El tipo de tratamiento se da por el tipo, etapa y grado específico del cáncer. La quimioterapia consiste en el uso de uno o más compuestos químicos (quimioterápicos) para el tratamiento del cáncer y de enfermedades causadas por agentes biológicos, y puede administrarse concomitantemente con cirugía y radioterapia. Conclusión: Es importante que el enfermero tenga conocimiento específico para actuar como educador en salud y prestador de la asistencia al paciente en tratamiento quimioterápico ambulatorial y para promoción de la relación interpersonal que proporcionará al paciente un enfrentamiento del tratamiento de manera más satisfactoria. Esta educación, orientada por la capacidad de comprensión de cada individuo, promueve el entendimiento correcto de la enfermedad, el tratamiento, el manejo de los efectos colaterales y las vivencias de los pacientes.

Palabras clave: Antineoplásicos, Quimioterapia, Cuidados de Enfermería.

\section{INTRODUÇÃO}

Segundo o Instituto Nacional de Câncer (INCA) (2018), o câncer abrange um grupo de mais de 100 doenças que possuem em comum a capacidade de crescimento irregular de suas células que acometem tecidos e órgãos, sendo capazes de atingir outras partes do corpo. As causas do câncer são diversas, podendo ser classificadas em externas ou internas ao organismo, estando ambas relacionadas umas às outras.

As causas externas estão ligadas ao meio ambiente e aos hábitos de vida de cada indivíduo. Já as causas internas, são, na maioria dos casos, genéticas, relacionando-se à eficácia do organismo de combater as agressões externas. Dos casos de cânceres, 80 a $90 \%$ estão ligados a fatores ambientais. O câncer pode surgir dependendo da intensidade e do tempo de exposição aos fatores que o provocam (INCA, 2018).

De acordo com dados divulgados pela Organização Mundial da Saúde (OMS) (2017), mais de 8,8 milhões de pessoas morrem de câncer no mundo, sobretudo em países de baixa ou média renda. Estima-se que no Brasil, no biênio de 2018-2019, a incidência seja de 600 mil novos casos de câncer, para cada um dos anos, exceto para o câncer de pele não melanoma (170 mil) (INCA, 2017).

Os cânceres com maior incidência são os de próstata (68 mil) em homens e mama (60 mil) em mulheres. Na região sudeste do país, foram estimados para 2018 cerca de 135 mil novos casos de câncer em homens e 137 mil em mulheres, dos quais em torno de 29 mil casos em homens e 28 mil em mulheres são no estado de Minas Gerais (INCA, 2017).

O tratamento do câncer consiste em três modalidades: cirurgia, radioterapia e quimioterapia. Podem ser empregadas simultaneamente para o tratamento das neoplasias malignas, diferenciando-se apenas na sua indicação e na importância de cada uma (BRASIL, 2016).

A preferência pelo tipo de tratamento para o câncer ocorre pelo tipo, estágio e grau específico do câncer. Os objetivos do tratamento são a de cura da doença maligna, sobrevida e controle das células neoplásicas, e diminuição dos sintomas relacionados à doença e melhoria na qualidade de vida. Não se inicia o tratamento até que o diagnóstico do câncer seja comprovado, além do esclarecimento de seu estadiamento e sua gradação, que está relacionado com a classificação das células tumorais (HINKLE JL e CHEEVER KH, 2017).

A quimioterapia consiste no uso de um ou mais compostos químicos (quimioterápicos) para o tratamento do câncer e de doenças causadas por agentes biológicos, podendo ser aplicada concomitante a cirurgia e a radioterapia. A quimioterapia pode ser classificada em: curativa, onde se consegue a eliminação completa 
do tumor; adjuvante, que se segue à cirurgia, a fim de eliminar células residuais e possíveis metástases; neoadjuvante, como complemento da cirurgia e/ou radioterapia, para redução parcial do tumor antes de uma cirurgia; paliativa, utilizada para melhora da qualidade de vida do paciente, quando não há possibilidade de cura do mesmo (INCA, 2018).

Grande parte dos antineoplásicos age de forma inespecífica, impactando tanto as células normais como as células de câncer. Dessa forma, células em divisão celular acelerada sofrem, em consequência da quimioterapia, com mais intensidade. Os efeitos colaterais da quimioterapia variam de acordo com a classe quimioterápica, sua dose e o intervalo de tempo entre os ciclos, dentre outros fatores (RODRIGUES AB e OLIVEIRA PP, 2016).

Alguns dos efeitos colaterais que podem ocorrer são a fraqueza, diarreia, perda de peso, aumento de peso (edema), feridas na boca (mucosite), queda de cabelos (alopecia) e outros pelos do corpo, enjoos, vômitos e tonteiras (INCA, 2018).

Conforme o Artigo 11, da Lei oㅜ 7.498 de 25 de junho de 1986, com relação às atividades e atribuições dos Enfermeiros, compete a este, privativamente todos os cuidados diretos aos pacientes com gravidade que gere risco de morte, executar intervenções complexas, que necessite de entendimento científico, e a aptidão em resolver questões de forma rápida. Além disso, de acordo com a Resolução do Conselho Federal de Enfermagem (COFEN) (1998), é de competência do Enfermeiro que trabalha com quimioterápico antineoplásico administrá-lo, elaborar protocolos para prevenção, tratamento e diminuição de efeitos colaterais nos pacientes em uso de quimioterapia, além de proporcionar ações de prevenção de riscos e agravos, através da educação dos pacientes e familiares, a fim de garantir maior qualidade de vida para o paciente.

A assistência de enfermagem especializada é de extrema importância para a melhoria biopsicossocialespiritual de um paciente oncológico. A implementação dos cuidados ao paciente com câncer exige do enfermeiro a pluralidade de conhecimento e flexibilidade na atuação e, quando estão associadas a uma boa sistematização da assistência, as ações de enfermagem ao paciente são determinantes para uma gerência de boa qualificação (GUIMARÃES RCR, et al., 2015).

A enfermagem faz-se de grande importância durante todo o tratamento quimioterápico, sendo os profissionais que acompanham continuamente o paciente e que fornecem orientações relacionadas ao tratamento a todos os envolvidos. Esta orientação é essencial na compreensão destes pacientes e familiares, sendo possível sanar as dúvidas e possibilitar uma melhor aderência ao tratamento quimioterápico e no enfrentamento diante da patologia (CUNHA FF, et al., 2017).

Portanto, o objetivo deste trabalho foi descrever os estudos que abordam os cuidados de enfermagem aos pacientes oncológicos adultos em tratamento quimioterápico sistêmico ambulatorial.

\section{MÉTODOS}

O presente estudo caracteriza-se como uma revisão integrativa, que é definida como uma forma de construir uma análise vasta da literatura, compreender sobre um assunto de acordo com pesquisas anteriores, bem como permitir o pensamento sobre uma pesquisa futura (MENDES KDS, et al., 2008).

Iniciou-se a pesquisa realizando um levantamento do acervo referente ao tema estudado a partir de publicações nas bases de dados: Scientific Electronic Library Online (SCIELO), Biblioteca Virtual em Saúde (BVS) e Literatura Latino-americana e do Caribe em Ciências da Saúde (LILACS). Os Descritores em Ciências da Saúde (DECS) da BVS selecionados foram: antineoplásicos; cuidados de enfermagem. Esses descritores conectados pelo operador booleano AND, nortearam a busca pelos estudos científicos, orientados pela seguinte pergunta de pesquisa: "Quais os cuidados de enfermagem necessários ao paciente oncológico adulto em tratamento quimioterápico sistêmico ambulatorial?".

Foi utilizado na SCIELO, português e inglês como filtro, e na BVS e LILACS, texto completo disponível, português e inglês. A coleta de dados e a análise dos resultados foram feitas nos dias 15 de maio a 30 de 
junho de 2018. Para inclusão dos artigos foram estabelecidos os seguintes critérios: publicações em português e inglês, com texto completo disponível, voltadas para o cuidado de enfermagem ao paciente oncológico adulto em tratamento quimioterápico sistêmico ambulatorial, sem utilizar um intervalo de tempo específico.

Os critérios de exclusão adotados foram: artigos que não atendessem o objetivo do estudo, voltados para pediatria e geriatria e direcionados a quimioterapia oral.

Após busca realizada e a seleção conforme os critérios de inclusão e exclusão tivemos a composição da amostra para o estudo. Foram selecionados para essa revisão integrativa 17 artigos. Os dados foram coletados, sintetizados e organizados a fim de que pudéssemos atingir o objetivo proposto em questão. Para maior clareza, segue Fluxograma 1 abaixo com os detalhes mencionados.

Fluxograma 1 - Processo metodológico sintetizado para seleção dos artigos científicos.

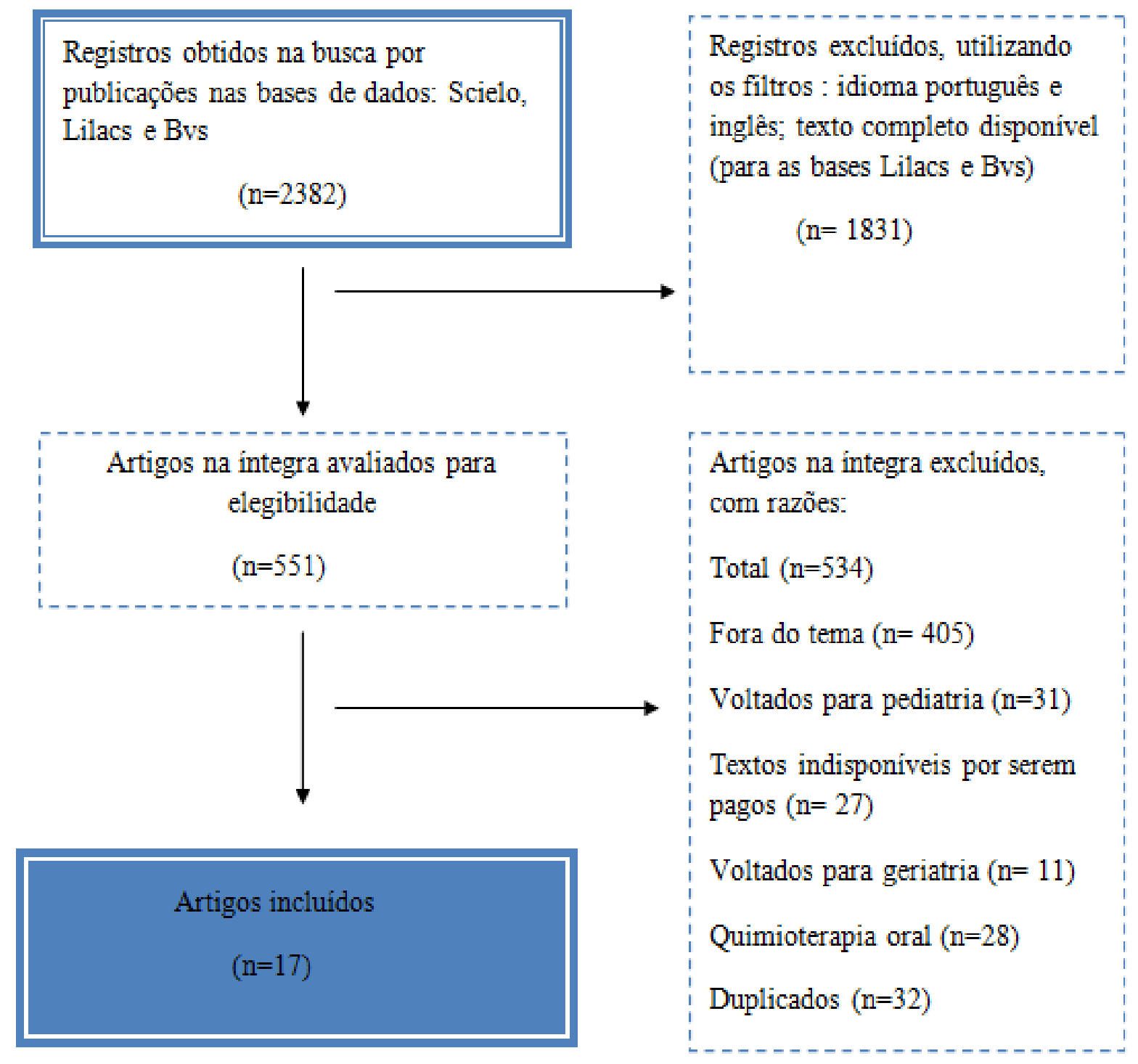

Fonte: Dados da pesquisa, 2018.

\section{RESULTADOS}

Seguem no Quadro 1 abaixo os artigos selecionados com base nos critérios de inclusão e exclusão adotados nesse estudo. 
Quadro 1- Seleção dos estudos para revisão integrativa.

\begin{tabular}{|c|c|c|c|c|c|}
\hline Título & Base & Periódico & Qualis & Tipo de pesquisa & Ano \\
\hline $\begin{array}{l}\text { Enfermagem à mulher em tratamento } \\
\text { quimioterápico - uma análise compreensiva } \\
\text { do assistir. }\end{array}$ & BVS & $\begin{array}{l}\text { Revista Brasileira } \\
\text { de Enfermagem }\end{array}$ & A2 & $\begin{array}{l}\text { Pesquisa de } \\
\text { campo: Qualitativa }\end{array}$ & $\begin{array}{l}199 \\
8\end{array}$ \\
\hline $\begin{array}{l}\text { Quimioterapia antineoplásica: revisão da } \\
\text { literatura }\end{array}$ & LILACS & \begin{tabular}{|l|} 
Revista Mineira de \\
Enfermagem \\
(REME)
\end{tabular} & B1 & $\begin{array}{l}\text { Revisão } \\
\text { literatura }\end{array}$ & $\begin{array}{l}199 \\
9\end{array}$ \\
\hline $\begin{array}{l}\text { A existencialidade da pessoa com neoplasia } \\
\text { em tratamento quimioterápico }\end{array}$ & LILACS & $\begin{array}{l}\text { Acta Scientiarum- } \\
\text { Health Sciences }\end{array}$ & B2 & $\begin{array}{l}\text { Pesquisa de } \\
\text { campo: Qualitativa }\end{array}$ & $\begin{array}{l}200 \\
3\end{array}$ \\
\hline $\begin{array}{l}\text { Monitoramento e avaliação dos efeitos } \\
\text { colaterais da quimioterapia em pacientes com } \\
\text { câncer de cólon }\end{array}$ & LILACS & \begin{tabular}{|lr} 
Revista & Latino- \\
Americana de & \\
Enfermagem & \\
\end{tabular} & A1 & $\begin{array}{l}\text { Pesquisa } \\
\text { campo: } \\
\text { Quantitativa }\end{array}$ & $\begin{array}{l}200 \\
4\end{array}$ \\
\hline $\begin{array}{l}\text { Quimioterapia antineoplásica e nutrição: uma } \\
\text { relação complexa }\end{array}$ & LILACS & $\begin{array}{l}\text { Revista Eletrônica } \\
\text { de Enfermagem }\end{array}$ & B1 & $\begin{array}{l}\text { Pesquisa } \\
\text { campo: } \\
\text { Quantitativa }\end{array}$ & $\begin{array}{l}200 \\
8\end{array}$ \\
\hline $\begin{array}{l}\text { A relação humana no cuidado de } \\
\text { enfermagem junto ao cliente com câncer } \\
\text { submetido a terapêutica antineoplásica }\end{array}$ & LILACS & $\begin{array}{l}\text { Acta Paulista de } \\
\text { Enfermagem }\end{array}$ & A2 & $\begin{array}{l}\text { Pesquisa de } \\
\text { campo: Qualitativa }\end{array}$ & $\begin{array}{l}200 \\
8\end{array}$ \\
\hline $\begin{array}{l}\text { Desenvolvimento de instrumento de coleta de } \\
\text { dados de enfermagem para pacientes com } \\
\text { câncer de pulmão em quimioterapia } \\
\text { ambulatorial }\end{array}$ & LILACS & $\begin{array}{ll}\text { Escola Anna Nery- } \\
\text { Revista } & \text { de } \\
\text { Enfermagem } & \end{array}$ & B1 & $\begin{array}{l}\text { Pesquisa de } \\
\text { campo: Qualitativa }\end{array}$ & $\begin{array}{l}200 \\
8\end{array}$ \\
\hline $\begin{array}{l}\text { Assistência de enfermagem a portadores de } \\
\text { linfoma de hodgkin submetidos à } \\
\text { quimioterapia: revisão integrativa }\end{array}$ & LILACS & \begin{tabular}{|lr} 
Online r & Brazilian \\
Journal of Nursing
\end{tabular} & B1 & $\begin{array}{l}\text { Revisão } \\
\text { integrativa }\end{array}$ & $\begin{array}{l}200 \\
8\end{array}$ \\
\hline $\begin{array}{l}\text { Aspectos a serem abordados por enfermeiros } \\
\text { na consulta a pacientes em uso de } \\
\text { quimioterápicos potencialmente neurotóxicos }\end{array}$ & BVS & \begin{tabular}{|l|} 
Revista de \\
Enfermagem \\
UFPE online
\end{tabular} & B2 & $\begin{array}{l}\text { Pesquisa de } \\
\text { campo: } \\
\text { Quantitativa } \\
\end{array}$ & $\begin{array}{l}201 \\
1\end{array}$ \\
\hline $\begin{array}{l}\text { Síndrome mão-pé induzida por quimioterapia: } \\
\text { relato de um caso }\end{array}$ & LILACS & \begin{tabular}{|l|}
$\begin{array}{l}\text { Revista Brasileira } \\
\text { de Enfermagem }\end{array}$ \\
\end{tabular} & A2 & Relato de caso & $\begin{array}{l}201 \\
2\end{array}$ \\
\hline $\begin{array}{l}\text { Náuseas, vômitos e qualidade de vida de } \\
\text { mulheres com câncer de mama em } \\
\text { tratamento quimioterápico }\end{array}$ & LILACS & $\begin{array}{l}\text { Revista Gaúcha } \\
\text { de Enfermagem }\end{array}$ & B1 & $\begin{array}{l}\text { Pesquisa } \\
\text { campo: } \\
\text { Quantitativa }\end{array}$ & $\begin{array}{l}201 \\
3\end{array}$ \\
\hline $\begin{array}{l}\text { Perfil sociodemográfico e clínico de pessoas } \\
\text { em tratamento quimioterápico: subsídios para } \\
\text { o gerenciamento em enfermagem }\end{array}$ & LILACS & $\begin{array}{l}\text { Revista Eletrônica } \\
\text { de Enfermagem }\end{array}$ & B1 & $\begin{array}{l}\text { Pesquisa } \\
\text { campo: } \\
\text { Quantitativa }\end{array}$ & $\begin{array}{l}201 \\
3\end{array}$ \\
\hline $\begin{array}{l}\text { The Effects of Education on Anxiety Levels in } \\
\text { Patients Receiving Chemotherapy for the First } \\
\text { Time: An Integrative Review }\end{array}$ & BVS & $\begin{array}{l}\text { Clinical Journal of } \\
\text { Oncology Nursing }\end{array}$ & A1 & rão & $\begin{array}{l}201 \\
4\end{array}$ \\
\hline $\begin{array}{l}\text { The Patient Remote Intervention and } \\
\text { Symptom Management System (PRISMS) - a } \\
\text { Telehealth mediated intervention enabling } \\
\text { real-time monitoring of chemotherapy side- } \\
\text { effects in patients with haematological } \\
\text { malignancies: study protocol for a randomized } \\
\text { controlled trial }\end{array}$ & BVS & Trials Journal & B1 & $\begin{array}{l}\text { Estudo randomiza } \\
\text { do } \\
\text { controlado }\end{array}$ & $\begin{array}{l}201 \\
5\end{array}$ \\
\hline $\begin{array}{l}\text { Nursing Intervention to Enhance Outpatient } \\
\text { Chemotherapy Symptom Management: } \\
\text { Patient-Reported Outcomes of a Randomized } \\
\text { Controlled Trial }\end{array}$ & BVS & Câncer & A1 & $\begin{array}{l}\text { Estudo randomiza } \\
\text { do controlado }\end{array}$ & $\begin{array}{l}201 \\
5\end{array}$ \\
\hline $\begin{array}{l}\text { A gerência do cuidado de enfermagem à } \\
\text { mulher com câncer de mama em } \\
\text { quimioterapia paliativa }\end{array}$ & SCIELO & $\begin{array}{l}\text { Texto \& Contexto - } \\
\text { Enfermagem }\end{array}$ & A2 & $\begin{array}{lr}\text { Pesquisa } & \text { de } \\
\text { campo: Qualitativa }\end{array}$ & $\begin{array}{l}201 \\
6\end{array}$ \\
\hline $\begin{array}{l}\text { Implementation of educational manuals in } \\
\text { nursing consultation: opinion of patients } \\
\text { submitted to antineoplastic chemotherapy. }\end{array}$ & BVS & $\begin{array}{l}\text { Journal of Nursing } \\
\text { UFPE online }\end{array}$ & B2 & $\begin{array}{l}\text { Pesquisa de } \\
\text { campo: Qualitativa }\end{array}$ & $\begin{array}{l}201 \\
7\end{array}$ \\
\hline
\end{tabular}

Fonte: Dados da pesquisa, 2018. 
A base de dados LILACS obteve dez artigos (58,83\%), revelando maior relevância no que tange o número de artigos encontrados. Com seis artigos (35,29\%) esteve a BVS, e a SCIELO com um artigo $(5,88 \%)$.

Ao avaliar o número de artigos publicados em cada revista, observaram-se duas publicações $(11,78 \%)$, na Revista Brasileira de Enfermagem e duas publicações (11,78\%), na Revista Eletrônica de Enfermagem.

As demais revistas, Texto \& Contexto - Enfermagem, Clinical Journal of Oncology Nursing, Revista de Enfermagem UFPE online, Journal of Nursing UFPE online, Trials Journal, Cancer, Revista Mineira de Enfermagem - REME, Acta Scientiarum - Health Sciences, Revista Latino-Americana de Enfermagem, Acta Paulista de Enfermagem, Escola Anna Nery - Revista de Enfermagem, Online Brazilian Journal of Nursing e Revista Gaúcha de Enfermagem, apresentaram cada uma, uma publicação $(5,88 \%)$.

Relacionado ao qualis dos periódicos, seis deles (40\%), apresentaram qualis B1, enquanto três (20\%), qualis A1, três (20\%), qualis A2 e três (20\%), qualis B2. Segue abaixo o Gráfico 1 com a distribuição dos periódicos relacionados ao qualis.

Gráfico 1- Representação do qualis dos periódicos.

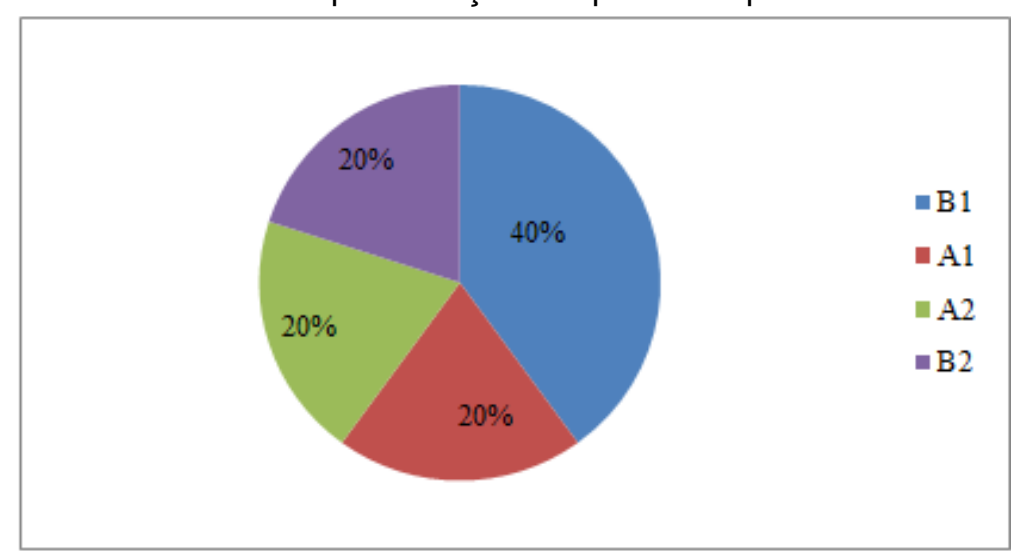

Fonte: Dados da pesquisa, 2018.

Gráfico 2- Número de estudos por ano de publicação.



Fonte: Dados da pesquisa, 2018. 
Quanto a localização dos periódicos, dois eram do Rio de Janeiro (13,33\%), dois de Pernambuco (13,33\%), dois de São Paulo (13,33\%), um de Minas Gerais (6,67\%), um de Santa Catarina (6,67\%), um do Paraná $(6,67 \%)$, um do Rio Grande do Sul (6,67\%), um do Distrito Federal (6,67\%), um de Goiás $(6,67 \%)$, um da Pensilvânia $(6,67 \%)$, um de Londres $(6,66 \%)$ e um da Geórgia $(6,66 \%)$. Quanto ao tipo de pesquisa, seis artigos $(35,29 \%)$ eram pesquisas qualitativas, cinco $(29,41 \%)$ quantitativas, duas $(11,76 \%)$ revisões integrativas, duas $(11,76 \%)$ estudos randomizados controlados, uma $(5,89 \%)$ revisão da literatura e uma $(5,89 \%)$ relato de caso. A distribuição dos estudos por ano de publicação é apresentada a seguir no Gráfico 2.

De acordo com o gráfico, pode-se perceber que os artigos selecionados se encontraram no período de 1998-2017, com ausência de publicações nos anos 2000, 2001, 2002, 2005, 2006, 2007, 2009 e 2010. Apenas uma publicação nos anos 1998, 1999, 2003, 2004, 2011, 2012, 2014, 2016 e 2017. Duas publicações em 2013 e 2015 e quatro publicações em 2008, ano mais relevante quanto ao número de publicações.

\section{DISCUSSÃO}

Importante ressaltar que a maior parte dos periódicos (40\%), apresentaram qualis B1, e o qualis mais baixo foi B2, demonstrando que os artigos selecionados possuem uma qualidade notável. O sistema que classifica os trabalhos científicos de programas de pós-graduação publicados possui como denominação qualisperiódicos. Anualmente ocorre a atualização das classificações, onde A1 indica qualidade mais elevada, posteriormente se seguem as demais: A2, B1, B2, B3, B4, B5 e C que indica qualidade zero (BRASIL, 2018).

A região sudeste brasileira apresentou maior número de publicações, totalizando cinco artigos $(29,41 \%)$. Há uma centralização da produção nas regiões Sudeste e Sul (SIDONE OJG, et al., 2016). Além disso, no presente estudo, o câncer de mama foi o mais prevalente, detectado em oito artigos (47,05\%) e, segundo o INCA, esse câncer é o mais predominante no mundo, sendo entre as mulheres mais frequente, especialmente na região Sudeste (ALVES KR, et al., 2011).

Segundo Maruthappu M, et al. (2016) foi descoberto que a crise econômica global ocorrida na época obtinha relação com o excesso de óbitos por câncer entre 2008 e 2010. Isso demonstra que em 2008 houve um aumento do número de câncer de forma excessiva, sendo o ano de maior número de publicações desse estudo, totalizando quatro artigos (23,53\%).

A fim de organizar as informações e facilitar no entendimento, discutimos as informações relevantes em nosso trabalho, que compreendem o objetivo, em dois tópicos: Importância das orientações de enfermagem durante o tratamento quimioterápico e Cuidados de enfermagem ao paciente oncológico em tratamento quimioterápico.

\section{Importância das orientações de enfermagem durante o tratamento quimioterápico:}

O enfermeiro é peça fundamental na educação dos pacientes e seus familiares, pois possui significativo papel que se facilita por conta da proximidade com o paciente (CIRILO JD, et al., 2016). Dessa forma, ele se responsabiliza pelas informações quanto ao tratamento, sobre os medicamentos específicos que serão utilizados e os possíveis efeitos colaterais por conta da quimioterapia.

Segundo Alves KR, et al. (2011) as informações fornecidas pelo enfermeiro geram um desvendar do tratamento e doença, um cuidado conduzido de forma certa e a instrução direcionada do paciente. Os pacientes e familiares com a orientação de enfermagem podem ter a capacidade de se responsabilizar pelas atitudes que promovem o enfrentar das dificuldades resultantes do tratamento.

Vasques $\mathrm{Cl}$, et al. (2008) destacam a importância do papel do enfermeiro na educação ao paciente e familiar, as dúvidas relacionadas à doença, efeitos colaterais, controle da toxicidade, infecções e os impactos decorrentes do tratamento. Há relatos ainda, que as atividades educacionais ao paciente devem ser individualizadas, além de serem responsáveis pelo plano de cuidados.

De acordo com Silva MM, et al. (2013) uma orientação bem realizada promove êxito no tratamento e previne maiores malefícios, sendo preciso avaliar a forma como as pessoas vivem, sua escolaridade, o que 
conhecem, conforme os ditos populares, para que compreendam o que é o tratamento, os efeitos esperados e os não esperados, assim, minimizando a carência do saber e maximizando a chance de domínio do regime de tratamento.

Para isso, a comunicação entre os profissionais de enfermagem e os pacientes é essencial para a boa adesão ao tratamento quimioterápico e esclarecimento de dúvidas. Conforme Cirilo JD, et al. (2016) a comunicação deve valorizar os meios verbais e não verbais. Dessa forma, cabe ao enfermeiro avaliar a forma de comunicação com o paciente. Três artigos (17,65\%) relataram como prevalência, entre os pacientes, o ensino fundamental incompleto. Assim sendo, a linguagem deve ser adequada para um entendimento melhor das intervenções propostas (ALVES KR, et al., 2011).

De acordo com Gozzo TO, et al. (2013) a educação deve ser feita com uma linguagem que se possa compreender. Além disso, a conversa com o paciente pode se dar através de telefonemas. Segundo Breen $\mathrm{S}$, et al. (2015), a telessaúde ajuda os enfermeiros no cuidado aos pacientes, pois em seu estudo estes relataram como satisfatórias as ligações. Portanto, o diálogo com o paciente promove uma vivência melhor do tratamento.

Conforme Cruz FOAM, et al. (2017), o enfermeiro se comunicar com o paciente é essencial. Esse relacionamento interpessoal minimiza tensões enfrentadas pelo paciente em tratamento quimioterápico (CIRILO JD, et al., 2016). Um bom relacionamento promove calma e possibilidade de vencer obstáculos, o que gera leveza no dia-a-dia. Para diminuição do estresse vivido pelo paciente, o enfermeiro é essencial (VASQUES Cl, et al., 2008).

Essa aproximação com o enfermeiro é importante para o paciente. Como relatou Sales CA, et al. (2003) o portador de neoplasia procura ações de boa vontade que envolvem o seu estado de doença e não somente a intervenção à doença e ao corpo. A relação do profissional enfermeiro com o paciente necessita de manifestações de afeto, para que haja uma construção de confiança entre eles. A amizade, respeito, carinho, entre outros, durante a quimioterapia produzem a confiança (FONTES CAS e ALVIM NAT, 2008).

Além do relacionamento interpessoal com o paciente, é necessário o contato com o familiar. É importante o enfermeiro determinar o suporte ao paciente e o estabelecimento do cuidador que ajudará o paciente nas situações que ele não conseguirá enfrentar sozinho, mediante o tratamento e progressão da doença (ALVES $\mathrm{KR}$, et al., 2011). Após o tratamento ambulatorial, os efeitos colaterais são enfrentados pelo paciente e seu familiar (CRUZ FOAM, et al., 2017). É essencial a família interagir para encarar as dificuldades decorrentes da doença (SALES CA, et al., 2003). Assim, o enfermeiro precisa considerar a família como colaboradora na efetuação dos cuidados (SILVA MM, et al., 2013).

\section{Cuidados de enfermagem ao paciente oncológico em tratamento quimioterápico:}

Antes da realização da quimioterapia, a consulta de enfermagem deve ser implementada, pois é a partir da avaliação do paciente que o enfermeiro será capaz de identificar os problemas e realizar os cuidados de enfermagem. Conforme Alves KR, et al. (2011) o enfermeiro da área oncológica se responsabiliza pelos efeitos da quimioterapia causados ao paciente quanto a identificação, intervenções, avaliações e monitoramento, através da consulta de enfermagem que é direcionada a todos os pacientes. Estes serão acolhidos dispondo dados para que o diagnóstico de enfermagem seja realizado, bem como as intervenções necessárias. Durante a consulta o enfermeiro avalia se será preciso o encaminhamento a outros profissionais.

De acordo com Cirilo JD, et al. (2016) durante a consulta, o enfermeiro deve dar atenção às lamentações da paciente, levantando os cuidados necessários, promovendo o autocuidado, principalmente no que tange as reações adversas, bem como promovendo qualidade de vida. É necessário que se monitore continuamente o paciente e avalie se as intervenções estão sendo eficazes no domínio dos efeitos (ALMEIDA EPM, et al., 2004).

Para isso, é imprescindível para a prática clínica em enfermagem, o conhecimento. É importante que os profissionais de enfermagem compreendam as características da patologia e dos indivíduos por essa acometidos, os mecanismos da doença, suas manifestações clínicas, seus efeitos colaterais e tratamentos, 
tal como as intervenções de enfermagem que podem ser realizadas (VASQUES CI, et al., 2008). Segundo Silva MM, et al. (2013) é necessário gerenciar o cuidado de forma humanizada, individual e integral.

Assim, durante a consulta de enfermagem, é necessário que o enfermeiro leve em consideração as necessidades dos sexos por gênero, para melhor aplicação de cuidados durante o tratamento. Tendo em vista que no presente estudo, doze artigos (70,59\%) obtiveram predomínio do sexo feminino, o enfermeiro deverá elaborar medidas que não prejudiquem as ações realizadas pela mulher referentes ao lar, ao trabalho e à família. Quanto aos pacientes do sexo masculino, o enfermeiro deve levar em consideração a sexualidade, o fisiológico e a posição do homem na sociedade (ALVES KR, et al., 2011).

Além disso, de acordo com Salvadori AM, et al. (2008) os pacientes que recebem quimioterápicos possuem necessidades físicas e emocionais, dúvidas relacionadas ao tratamento, que conforme Garcia S (2014), a ansiedade quanto a essas questões pode ser diminuída através da educação ao paciente. Segundo Alves $\mathrm{KR}$, et al. (2011) é necessário que o enfermeiro, identifique os problemas psicossociais, físicos, mentais e espirituais e proponha cuidados que melhorem a vida dos pacientes.

O enfermeiro deve ser capaz de observar as limitações físicas de seus pacientes, como as pessoas em estágio evoluído do câncer, para que ele providencie um ambiente confortável, avaliando a necessidade de a pessoa manter-se em cadeira de rodas ou maca enquanto está no ambulatório (SILVA MM, et al., 2013).

Além da consulta de enfermagem, é necessária a realização da triagem antes da administração do quimioterápico, pois dessa forma, o enfermeiro é capaz de observar tudo aquilo que possa impossibilitar a administração no dia marcado. Porém, esse impeditivo deve ser avaliado com cuidado, pois o paciente acredita que irá receber o tratamento e, uma vez que ele não se trata, os sentimentos negativos podem aparecer (CIRILO JD, et al., 2016).

Para que o paciente esteja apto para dar início à quimioterapia, é necessário que ele atenda a determinados requisitos, devendo possuir perda de peso inferior a 10\%, tolerar clinicamente as medicações quimioterápicas, não possuir infecção ou que esta esteja controlada, e estar com as células sanguíneas e dosagens séricas dentro do padrão aceitável (RIUL S e AGUILLAR OM, 1999).

Além disso, a avaliação do paciente é feita conforme os índices de Zubrod e Karnofsky, que se relacionam aos sintomas apresentados. Com relação às contraindicações absolutas dos quimioterápicos aos pacientes oncológicos, elas se destinam a pacientes em fase terminal, ou com infecções graves, os que estão em coma e gestantes no primeiro trimestre. É relativo receber o quimioterápico, pacientes com idade menor que três meses e maior que 80 anos, metástase cerebral com impossibilidade de cirurgia ou que não responda a radioterapia, comprometimento grave de outros órgãos vitais, falta de colaboração do paciente em aderir ao tratamento, o que o impossibilita de recebê-lo e os casos em que o tumor não reage as medicações quimioterápicas propostas (RIUL S e AGUILLAR OM, 1999).

Além de conhecer os critérios para se administrar a quimioterapia, o enfermeiro necessita conhecer os antineoplásicos. Nesse estudo, sete artigos $(41,18 \%)$ citaram antineoplásicos, sendo mais mencionado o fluorouracil (Flusan $\AA$ ), em cinco destes artigos. De acordo com Vasques $\mathrm{Cl}$, et al. (2008) o enfermeiro precisa conhecer as modalidades de tratamento para que se desenvolva a intervenção de enfermagem aos pacientes.

Durante o tratamento quimioterápico, o enfermeiro precisa orientar o paciente a se abster de substâncias que possam prejudicar o tratamento, como o tabaco e o álcool. Segundo Alves KR, et al. (2011), o tabaco é uma das causas de várias doenças, como as cardiovasculares, respiratórias e o câncer. Além disso, Salvadori AM, et al. (2008) referiram, em seu estudo sobre câncer no pulmão, que a maioria (95\%) dos participantes, declararam-se tabagistas ou já terem sido. Conforme Alves KR, et al. (2011) o uso do álcool durante o tratamento antineoplásico é impactante, uma vez que atinge os nervos periféricos, sendo necessário alertar os pacientes quanto ao álcool na fase de tratamento.

$\mathrm{O}$ enfermeiro deve atentar quanto à gravidez das pacientes em tratamento. Nesse estudo, doze artigos (70,59\%) abordaram pacientes do sexo feminino e quatro artigos $(23,53 \%)$ apresentaram mulheres com possibilidade de engravidar, onde um deles apresentou a idade de 37 anos, um com intervalo de 29-50 anos 
e dois com intervalo de 41-50 anos. Conforme Alves KR, et al. (2011) é importante orientar a mulher em tratamento quimioterápico a evitar a gravidez, uma vez que existem mulheres que podem engravidar e fazem quimioterapia.

Os autores Riul S e Aguillar OM (1999) e Salvadori AM, et al. (2008) descreveram a importância de orientar quanto à fertilidade e métodos de contracepção e a importância da necessidade de se evitar a gravidez durante o tratamento quimioterápico, por conta dos efeitos teratogênicos dos antineoplásicos e possíveis abortos que os antineoplásicos podem ocasionar.

Além desses cuidados necessários de serem realizados pelo enfermeiro, conhecer os efeitos que o tratamento quimioterápico gera nos pacientes é de suma importância para o estabelecimento dos cuidados de enfermagem. Uma vez que a enfermagem identifica os problemas dos pacientes, sua assistência pode gerar minimização dos sintomas (SALVADORI AM, et al., 2008).

Uma das situações que impactam o paciente em tratamento quimioterápico é a licença médica, pois pode atingir seu fator econômico e da família, visto que o valor recebido pela previdência é menor que o recebido no trabalho anterior. Essa condição pode colaborar na ansiedade, diminuição da qualidade de vida $\mathrm{e}$ comprometimento na adesão aos cuidados (ALVES KR, et al., 2011).

Três artigos (17,64\%) apresentaram a condição socioeconômica dos pacientes. A licença médica, o trabalho doméstico e o baixo nível socioeconômico foram apresentados cada um em um desses artigos. Assim, torna-se evidente o cuidado que o enfermeiro deve prestar ao paciente mediante tal situação. $O$ enfermeiro pode apresentar atividades laborais e biblioterapia para a melhora do emocional e da saúde mental (ALVES KR, et al., 2011). Conforme Silva MM, et al. (2013) a prática laborativa ajuda na autoestima. Outra situação vivenciada pelo paciente é a rede venosa danificada. A quimioterapia prejudica as veias (ALMEIDA EPM, et al., 2004). Por esse motivo há necessidade do aumento da ingesta hídrica para essa situação. Assim, torna-se necessário orientar o paciente a aumentar a ingesta hídrica. Camargo TC e Souza IEO (1998) descreveram que o enfermeiro deve verificar a necessidade de uso de acessos venosos centrais, levando em consideração o PICC como solução.

A alimentação do paciente é outro fator que deve ser considerado, uma vez que no estudo de Ferreira NMLA, et al. (2008) foi detectado a diminuição do consumo de alimentos, priorização pelos pacientes por aqueles mais macios e líquidos, descartando feijão, massas, carnes, leite e derivados, optando por sucos, verduras, vegetais e frutas. No estudo de Sales CA, et al. (2003) foi verificado que a quimioterapia colabora na mudança alimentar.

Almeida EPM, et al. (2004) apontaram a necessidade de informar quanto ao tipo de alimentos indicados e não indicados, a dieta e limpeza dos alimentos. É importante que o enfermeiro observe o paciente quanto a indícios de alterações nutricionais evidenciadas na pele e mucosas, como o ressecamento e desidratação (FERREIRA NMLA, et al., 2008).

O vômito é outro problema decorrente do tratamento quimioterápico. Almeida EPM, et al. (2004) declararam que os antieméticos estão no protocolo, porém o paciente decide se irá usá-lo, mas é necessário orientar para que não se agrave a situação, pois isso pode levar o paciente a não continuar com o tratamento. O profissional enfermeiro necessita conceder os antieméticos de forma que o paciente utilize o tanto necessário no momento mais complicado e deve investigar a capacidade do paciente em usar a medicação. Orientar quanto a alimentos frios, pois possuem maior aceitação, uma vez que os alimentos quentes possuem um odor que incomoda o paciente, orientando, ainda, quanto à ausência de gorduras (VASQUES Cl, et al., 2008).

Outro efeito colateral gastrintestinal é a diarreia, que pode ser vivenciada pelo paciente em tratamento de quimioterapia, porém, o antidiarreico não é prescrito como medicação profilática. Assim, a enfermagem necessita orientar quanto a ingesta hídrica e determinado tipo de dieta, a fim de diminuir esse efeito. Além disso, há a necessidade de higienização das regiões perianal e perineal (ALMEIDA EPM, et al., 2004). Vasques $\mathrm{Cl}$, et al. (2008) apresentaram a medicação kaopectato (KAOPECTATO 197.2 MG/ ML + 4.4 MG/ 
ML SUSPENSÃO ORAL) como um apoio para o manejo da diarreia. Esses mesmos autores relataram outro efeito colateral relacionado ao sistema gastrintestinal, a constipação, onde uma alimentação abundante em fibras se torna eficiente contra esse efeito. Segundo Gozzo TO, et al. (2013) informar quanto ao tratamento e a forma de lidar com seus efeitos indesejáveis é importante para administrar as toxinas do trato gastrintestinal.

A alopecia, caracterizada pela queda de cabelo, é muito abordada pelos pacientes, por prejudicar o relacionamento com as pessoas (SALES CA, et al., 2003). Nesse sentido, Vasques Cl, et al. (2008) declararam que o enfermeiro precisa orientar o paciente que o cabelo crescerá novamente, recomendando 0 corte mediante as quedas de cabelo e uso de perucas. Caso o paciente queira permanecer com seu cabelo, o torniquete de pressão ou capa gelada podem ser usados, mas, esclarecer quanto à alopecia, pode conduzir o paciente a vivenciar a situação da melhor maneira.

Outro efeito colateral que o paciente em tratamento com quimioterápico pode vivenciar é a síndrome mãopé. Conforme Simão DAS, et al. (2012) os pacientes em tratamento quimioterápico com mãos e pés com edema, vermelhidão e referindo dor, podem receber o diagnóstico da síndrome. O enfermeiro deve orientar quanto ao uso do creme de aloe vera neutro, aplicado três vezes por dia, manipulado segundo o retorno venoso, associado a uma alimentação de três em três horas com alimentos de cores vastas, e ingesta hídrica de, no mínimo, dois litros por dia, o que promove a melhora da síndrome.

Além desses efeitos, Vasques $\mathrm{Cl}$, et al. (2008) relataram que o risco de infecção quando há a manifestação de mucosite e estomatite, pode ser minimizado ao usar escovas macias, uso de antibióticos e antifúngicos de forma certa, bem como enxaguante bucal com ausência de álcool, sendo de clorexidina. Além disso, os mesmos autores declararam que a quimioterapia pode levar a fibrose pulmonar, sendo necessário realizar a ausculta do pulmão, radiografias e avaliar o padrão respiratório.

\section{CONCLUSÃO}

Foi identificada a necessidade de o enfermeiro buscar conhecimento aprofundado sobre a área oncológica, uma vez que nesse estudo verificou-se a relevância deste profissional enquanto educador em saúde no tratamento quimioterápico. Além disso, o enfermeiro deve realizar um cuidado individualizado avaliando as necessidades próprias de cada paciente, segundo os sintomas revelados. Por fim, foi percebida na pesquisa a ausência de cuidados de enfermagem quanto a outras questões como: intervenções relacionadas ao fluxo menstrual da mulher, fraqueza, tonteira, cuidado ao fazer a barba e a unha, que são necessários. Assim, é preciso mais estudos sobre os cuidados de enfermagem aos pacientes oncológicos em tratamento ambulatorial.

\section{REFERÊNCIAS}

1. ALMEIDA EPM, et al. Monitoramento e avaliação dos efeitos colaterais da quimioterapia em pacientes com câncer de cólon. Revista Latino-Americana de Enfermagem, 2004; 12(5): 760-766.

2. ALVES KR, et al. Aspectos a serem abordados por enfermeiros na consulta a pacientes em uso de quimioterápicos potencialmente neurotóxicos. Revista de Enfermagem UFPE online, 2011; 5(6): 1423-1430.

3. BRASIL. Lei no 7.498 , de 25 de junho de 1986. Dispõe sobre a regulamentação do exercício da enfermagem, e dá outras providências. Brasília, DF, 1 de agosto 2018. Seção 1, p. 4.

4. BRASIL. 2018. Classificação da produção intelectual. Fundação CAPES. Disponível em: http://www.capes.gov.br/avaliacao/instrumentos-de-apoio/classificacao-da-producao-intelectual. Acesso em: $01 \mathrm{de}$ agosto de 2018.

5. BRASIL. Manual de bases técnicas da oncologia- SAI/SUS- Sistema de Informações Ambulatoriais. 23. ed. Brasília: Ministério da Saúde, 2016.

6. BRASIL. Resolução COFEN-210 de1998. Dispõe sobre a atuação dos profissionais de Enfermagem que trabalham com quimioterápicos antineoplásicos. Rio de Janeiro, RJ, 1 de agosto 2018. Seção 1, p.4.

7. BREEN S, et al. The Patient Remote Intervention and Symptom Management System (PRISMS) - a Telehealth mediated intervention enabling real-time monitoring of chemotherapy side-effects in patients with haematological malignancies: study protocol for a randomised controlled trial. Trials, 2015; 16(472): 1-17.

8. HINKLE JL, CHEEVER KH. Tratado de enfermagem médico - cirúrgico. Rio de Janeiro: Guanabara Koogan, 2017; $788 p$. 
9. CAMARGO TC, SOUZA IEO. Enfermagem à mulher em tratamento quimioterápico - uma análise compreensiva do assistir. Revista Brasileira de Enfermagem, 1998; 51(3): 357-368.

10. CIRILO JD, et al. A gerência do cuidado de enfermagem à mulher com câncer de mama em quimioterapia paliativa. Texto \& Contexto Enfermagem, 2016; 25(3): 1-9.

11. CRUZ FOAM, et al. Implementation of educational manuals in nursing consultation: opinion of patients submitted to antineoplastic chemotherapy. Journal Nursing UFPE online, 2017; 11(5): 1757-1762.

12. CUNHA FF, et al. Representações de pacientes oncológicos sobre o tratamento de quimioterapia antineoplásica. Revista Fundamental Care Online, 2017; 9(3): 840-847.

13. FERREIRA NMLA, et al. Quimioterapia antineoplásica e nutrição: uma relação complexa. Revista Eletrônica de Enfermagem, 2008; 10(4): 1026-1034.

14. FONTES CAS, ALVIM NAT. A relação humana no cuidado de enfermagem junto ao cliente com câncer submetido à terapêutica antineoplásica. Acta Paulista de Enfermagem, 2008; 21(1): 77-83.

15. GARCIA S. The Effects of Education on Anxiety Levels in Patients Receiving Chemotherapy for the First Time: An Integrative Review. Clinical Journal of Oncology Nursing, 2014; 18(5): 516-521.

16. GOZZO TO, et al. Náuseas, vômitos e qualidade de vida de mulheres com câncer de mama em tratamento quimioterápico. Revista Gaúcha de Enfermagem, 2013; 34(3): 110-116.

17. GUIMARÃES RCR, et al. Ações de enfermagem frente às reações a quimioterápicos em pacientes oncológicos. Revista de Pesquisa Cuidado é Fundamental Online, 2015; 7(2): 2440-2452.

18. INSTITUTO NACIONAL DE CÂNCER JOSÉ ALENCAR GOMES DA SILVA, 2017.

19. INSTITUTO NACIONAL DE CÂNCER JOSÉ ALENCAR GOMES DA SILVA, 2018.

20. MARUTHAPPU M, et al. Economic downturns, universal health coverage, and cancer mortality in high-income and middle-income countries, 1990-2010: a longitudinal analysis. The Lancet, 2016; 388(10045): $684-695$.

21. MENDES KDS, et al. Revisão integrativa: método de pesquisa para a incorporação de evidências na saúde e na enfermagem. Texto \& Contexto Enfermagem, 2008; 17(4): 758-64.

22. RIUL S, AGUILLAR OM. Quimioterapia antineoplásica: revisão da literatura. Revista Mineira de Enfermagem, 1999; $3(1 / 2): 60-67$.

23. RODRIGUES AB, OLIVEIRA PP. Oncologia para enfermagem. São Paulo: Manole, 2016; 528p.

24. SALES CA, et al. A existencialidade da pessoa com neoplasia em tratamento quimioterápico. Acta Scientiarum- Health Sciences, 2003; 25(2): 177-182.

25. SALVADORI AM, et al. Desenvolvimento de instrumento de coleta de dados de enfermagem para pacientes com câncer de pulmão em quimioterapia ambulatorial. Escola Anna Nery- Revista de Enfermagem, 2008; $12(1)$ : $130-135$.

26. SIDONE OJG, et al. A ciência nas regiões brasileiras: evolução da produção e das redes de colaboração científica. Revista TransInformação, 2016; 28(1): 15-31.

27. SILVA MM, et al. Perfil sociodemográfico e clínico de pessoas em tratamento quimioterápico: subsídios para o gerenciamento em enfermagem. Revista Eletrônica de Enfermagem, 2013; 15(3): 704-712.

28. SIMÃO DAS, et al. Síndrome mão-pé induzida por quimioterapia: relato de um caso. Revista Brasileira de Enfermagem, 2012; 65(2): 374-378.

29. TRAEGER L, et al. Nursing Intervention to Enhance Outpatient Chemotherapy Symptom Management: PatientReported Outcomes of a Randomized Controlled Trial. Cancer, 2015; 121(21): 3905-3913.

30. VASQUES CI, et al. Assistência de enfermagem a portadores de linfoma de hodgkin submetidos à quimioterapia: revisão integrativa. Online Brazilian Journal of Nursing, 2008; 7(1): 1-9.

31. OMS. 2017. Early cancer diagnosis saves lives, cuts treatment costs. Disponível em: http://www.who.int/mediacentre/news/releases/2017/early-cancer-costs/en/. Acesso em: 15 nov. 2017. 